\title{
Exploring Phenolic Compounds as Quorum Sensing Inhibitors in Foodborne Bacteria
}

\section{Catarina Angeli Santos, Emília Maria França Lima, Bernadette Dora Gombossy de Melo Franco and Uelinton Manoel Pinto*}

Department of Food and Experimental Nutrition, Food Research Center, Faculty of Pharmaceutical Sciences, University of São Paulo, São Paulo, Brazil

The emergence of multidrug-resistant bacteria stimulates the search for new substitutes to traditional antimicrobial agents, especially molecules with antivirulence properties, such as those that interfere with quorum sensing (QS). This study aimed to evaluate the potential of phenolic compounds for QS inhibition in a QS biosensor strain (Chromobacterium violaceum) and three foodborne bacterial species (Aeromonas hydrophila, Salmonella enterica serovar Montevideo, and Serratia marcescens). Initially, an in silico molecular docking study was performed to select the compounds with the greatest potential for QS inhibition, using structural variants of the CviR QS regulator of $C$. violaceum as target. Curcumin, capsaicin, resveratrol, gallic acid, and phloridizin presented good affinity to at least four CviR structural variants. These phenolic compounds were tested for antimicrobial activity, inhibition of biofilm formation, and anti-QS activity. The antimicrobial activity when combined with kanamycin was also assessed. Curcumin, capsaicin, and resveratrol inhibited up to $50 \%$ of violacein production by $C$. violaceum. Biofilm formation was inhibited by resveratrol up to $80 \%$ in $A$. hydrophila, by capsaicin and curcumin up to $40 \%$ in S. Montevideo and by resveratrol and capsaicin up to $60 \%$ in S. marcescens. Curcumin completely inhibited swarming motility in S. marcescens. Additionally, curcumin and resveratrol increased the sensitivity of the tested bacteria to kanamycin. These results indicate that curcumin and resveratrol at concentrations as low as $6 \mu \mathrm{M}$ are potential quorum sensing inhibitors besides having antimicrobial properties at higher concentrations, encouraging applications in the food and pharmaceutical industries.

Keywords: quorum sensing, phenolics, antivirulence, antimicrobial, antibiofilm

\section{INTRODUCTION}

The microbial communication system called quorum sensing (QS) is used by a wide variety of bacteria allowing them to collectively modify their behavior in response to changes in cell density. This communication is mediated by small molecules accumulated during microbial multiplication and involves the production, secretion, and detection of extracellular signaling molecules, known as autoinducers (AI; Mukherjee and Bassler, 2019).

Gram-positive and Gram-negative bacteria exhibit different communication systems to regulate many physiological traits. In Gram-negative bacteria, signaling is usually mediated by acyl 
homoserine lactone (AHL) molecules, also known as autoinducer-1 (AI-1; Vanetti et al., 2020). In Gram-positive organisms, communication is mediated by autoinducer peptides which are usually secreted by ABC-type carrier proteins (Monnet et al., 2014; Lima et al., 2020).

In addition to these main QS autoinducers, the furanosyl borate diester, also known as autoinducer-2 (AI-2), is associated with both Gram-positive and Gram-negative bacteria allowing intra and interspecific communications (Chen et al., 2002). The autoinducer-3 (AI-3), a metabolite involved in pathogenesis of enterohemorrhagic Escherichia coli (EHEC), was recently elucidated and characterized (Kim et al., 2020). In fact, AI-3 analogs belong to the pyrazinone family, and they are derived from threonine dehydrogenase (Tdh) products and "abortive" tRNA synthetase reactions, being present in a variety of Gramnegative and Gram-positive pathogens (Kim et al., 2020). A number of other extracellular bacterial metabolites that function as signals in a range of microorganisms have also been discovered recently (Vanetti et al., 2020).

The genes and functions regulated by QS are diverse and can be classified into four groups: (1) cellular behavior, such as biofilm formation and dispersion, motility, and adhesion; (2) cell maintenance and proliferation, such as exoenzyme and siderophore synthesis, sporulation, and acid resistance; (3) horizontal gene transfer, such as conjugation in Agrobacterium tumefaciens; and (4) interactions with the host and other microorganisms, such as production of virulence factors, antibiotics and exo-polysaccharides, and bioluminescence, among others (Jimenez et al., 2010; Monnet et al., 2014; Grandclément et al., 2016; Lima et al., 2020).

The communication mediated by QS can be interrupted in several ways: by inhibiting the autoinducer synthesis, through enzymatic degradation of autoinducers, or by competition for binding to receptor proteins, ultimately inhibiting the target gene expression, mediated by interfering molecules called quorum sensing inhibitors (QSI; Zhang and Li, 2015; Grandclément et al., 2016).

Plants are one of the main sources of natural QSI, including medicinal plants, vegetables, and edible fruits. Some compounds derived from the secondary metabolism of plants significantly increase their ability to adapt to unfavorable environments. These metabolites, such as alkaloids, phenols, flavonoids, quinones, tannins, terpenes, and lecithins, are well-known defense mechanisms against herbivores and microorganisms (Nazir et al., 2020). Phenolic compounds are the second largest family of plant nutraceuticals, after terpenoids (Gutiérrez-Del-Rio et al., 2018). Recent studies have shown that phenolic compounds in extracts of edible foods can act as QSI (Quecán et al., 2019; Rivera et al., 2019; Santos et al., 2020).

Food spoilage causes great concern to the food industry as it leads to significant economic losses. The detection of QS signaling molecules in spoiled foods, as well as microbial interactions in fermentation of foods, has added a new dimension to the understanding of the spoilage process (Bai and Rai, 2011; Nazzaro et al., 2013; Martins et al., 2018; Almeida et al., 2020). Interference in QS communication could be an additional target to delay bacterial food spoilage since some phenotypes regulated by QS are also related to food deterioration, such as production of pectinases, lipases and proteases, and biofilm formation.

Antimicrobial resistance is one of the greatest threats to global health (WHO, 2018), and new alternatives are needed to treat infections and stop the spread of multidrug-resistant bacteria (Singh et al., 2019). The rationale for using QS inhibitors as an anti-virulence strategy is the lower selective pressure when compared to traditional antibiotics (Maura et al., 2016; Saeki et al., 2020).

This study evaluated the ability of some phenolic compounds to inhibit bacterial quorum sensing regulated phenotypes. First, we applied molecular docking with the CviR QS regulator of C. violaceum in order to select potential QSI, and then, we tested these compounds against several quorum sensingregulated phenotypes.

\section{MATERIALS AND METHODS}

\section{In silico Analysis - Selection of Phenolic Compounds by Molecular Docking}

Docking studies were performed according to Almeida et al. (2016, 2018). The potential anti-QS activity of 79 pre-selected compounds present in foods and plants, likely having antimicrobial and anti-QS activity (Supplementary Table 1), was evaluated, using six structural variants of the CviR QS regulator of $C$. violaceum. The crystallized structures of CviR (3QP1, 3QP2, 3QP4, 3QP5, 3QP6, and 3QP8) with different AHLs (Chen et al., 2011) were obtained from the RCSB Protein Data Bank database. ${ }^{1}$ The molecular structure of phenolic compounds, homoserine lactones, and furanones was obtained from the PubChem database. ${ }^{2}$ Molecular docking was performed using the "Dock Ligands" tool of the CLC Drug Discovery Workbench 4.0 software as described previously (Almeida et al., 2016, 2018). The five best docking scores of each compound were selected, allowing the inspection of the binding sites to the CviR protein.

\section{Phenolic Compound Preparation}

The next steps of the study were performed with the five phenolic compounds that presented the greatest inhibitory potential in in silico analyses: curcumin (PubChem CID: 969516), capsaicin (PubChem CID: 1548943), gallic acid (PubChem CID: 370), resveratrol (PubChem CID: 445154), and phloridizin (PubChem CID: 4789). The compounds (Sigma-Aldrich, Brazil) were dissolved in dimethyl sulfoxide (DMSO), so that the final solvent concentration did not exceed $1 \%$ in the tests (Rivera et al., 2019). Negative control comprised DMSO at the same concentration as in the tests with phenolic compounds.

\section{Bacterial Strains and Growth Conditions}

QS biosensor and foodborne bacterial species used in this study were Chromobacterium violaceum ATCC 12472,

${ }^{1}$ http://www.rcsb.org

${ }^{2}$ http://pubchem.ncbi.nlm.nih.gov 
Chromobacterium violaceum 026 and Serratia marcescens MG1, cultivated at $30^{\circ} \mathrm{C}$, as well as Aeromonas hydrophila IOC/FDA 110-36 and Salmonella Montevideo 163 (Monte et al., 2019), cultivated at $37^{\circ} \mathrm{C}$. All strains were grown in Luria-Bertani (LB) broth (KASVI) for $18 \mathrm{~h}$.

\section{Antimicrobial Activity - Minimal Inhibitory Concentration}

The antimicrobial activity of the selected phenolic compounds was evaluated by determination of the minimal inhibitory concentration (MIC), using the broth microdilution assay according to Wiegand et al. (2008), with some modifications. LB broth $(100 \mu \mathrm{l})$ containing different concentrations of each phenolic compound was added to a 96-well microtiter plate, and each well was inoculated with an overnight culture of the tested microorganism, adjusted to contain $10^{5} \mathrm{CFU} / \mathrm{ml}$. The controls were bacterial cultures in LB broth without the compounds, LB broth with each compound in each tested concentration without bacteria (color control), and LB broth (sterility control). Growth curves were determined by measuring the optical density at $595 \mathrm{~nm}\left(\mathrm{OD}_{595}\right)$ every $2 \mathrm{~h}$, during $12 \mathrm{~h}$ on a spectrophotometer (Multiskan FC, Thermo Fisher Scientific, Waltham, Massachussetts, United States). The MIC was the lowest concentration of the compound in which there was no bacterial growth, as observed by the growth curves. All QS assays were performed in concentrations that did not interfere with bacterial growth as recommended by Defoirdt et al. (2013).

\section{Anti-QS Activity}

\section{Violacein Production}

Quantification of violacein production was performed according to Santos et al. (2020), with modifications. The assay was performed in a 96-well microtiter plate containing $100 \mu \mathrm{l}$ of LB broth with each phenolic compound at sub-MIC and $10 \mu \mathrm{l}$ of inoculum containing $10^{6} \mathrm{CFU} / \mathrm{ml}$ of $C$. violaceum. The plates were incubated for $24 \mathrm{~h}$ at $30^{\circ} \mathrm{C}$ at $150 \mathrm{rpm}$ and then dried at $50^{\circ} \mathrm{C}$ in a BOD incubator. Subsequently, $100 \mu \mathrm{l}$ of pure DMSO was added to each well. After $30 \mathrm{~min}$ at room temperature, the $\mathrm{OD}_{595}$ was measured using a spectrophotometer (Multiskan FC, Thermo Fisher Scientific, Waltham, Massachussetts, United States). For the C. violaceum 026 assay, the autoinducer C6-HSL at $100 \mu \mathrm{M}$ was added to each well. LB broth without phenolic compounds was used as negative control. Results were expressed as percentages, comparing $\mathrm{OD}_{595}$ measurements for the tested phenolic compound and the negative control, which was considered $100 \%$.

\section{Swarming and Swimming Motility}

The test was performed according to Packiavathy et al. (2014) with some modifications. Swarming motility was tested using $3 \mathrm{ml}$ of semi-solid LB agar $0.5 \%(\mathrm{w} / \mathrm{v})$ to which the phenolic compounds were added at sub-MIC, and swimming motility was tested using $6 \mathrm{ml}$ of semi-solid LB agar $0.3 \%(\mathrm{w} / \mathrm{v})$ with the phenolic compounds at sub-MIC. These tests were performed in tubes containing melted semi-solid LB agar mixed with the tested phenolic compound. After vortexing, the mixtures were transferred to small Petri dishes $(49 \mathrm{~mm} \times 12 \mathrm{~mm})$. After $10 \mathrm{~min}$, $2 \mu \mathrm{l}$ of a culture inoculum containing $10^{8} \mathrm{CFU} / \mathrm{ml}$ of $A$. hydrophila or S. marcescens was spotted at the center of the plate, followed by $24 \mathrm{~h}$ incubation at the optimum growth temperature of the microorganism. Semi-solid agar without phenolic compound was used as control of absence of inhibition. The results of swarming and swimming motility inhibition were obtained comparing the bacterial growth diameters in the test and control plates.

\section{Biofilm Formation}

The analysis was performed according to Santos et al. (2020), using S. marcescens MG1, A. hydrophila, and S. Montevideo as target microorganisms. In a 96-well plate, $200 \mu \mathrm{l}$ of LB broth with the phenolic compound at sub-MIC was mixed with an aliquot of $20 \mu \mathrm{l}$ of an overnight culture of each tested bacterium, adjusted according to McFarland 0.5 solution $\left(10^{5} \mathrm{CFU} / \mathrm{ml}\right)$. The plates were incubated for $24 \mathrm{~h}$ at the optimal growth temperature of each bacterium. After removal of the culture medium, the plates were washed three times with $200 \mu \mathrm{l}$ of sterile saline $0.9 \%(\mathrm{w} / \mathrm{v})$. The sessile cells were stained with $200 \mu \mathrm{l}$ of crystal violet $0.1 \%(\mathrm{w} / \mathrm{v})$ for $30 \mathrm{~min}$, the dye was removed, and the wells were rinsed three times with saline solution. Each test included a negative control, correspondent to LB broth without the addition of phenolic compounds. The crystal violet retained by the adhered cells was dissolved in $200 \mu \mathrm{l}$ of $95 \%$ ethanol, and the $\mathrm{OD}_{595} \mathrm{~nm}$ was determined by spectrophotometry (Multiskan FC, Thermo Fisher Scientific, Waltham, Massachussetts, United States). Results were expressed as percentages, comparing $\mathrm{OD}_{595}$ measurements for the tested phenolic compound and the negative control, which was considered $100 \%$.

\section{Sensitivity to Kanamicin}

The MIC and minimum bactericidal concentration (MBC) of kanamycin (Sigma-Aldrich, Brazil) were determined using the broth microdilution method (CLSI, 2007). In a 96-well plate, $100 \mu \mathrm{l}$ of LB broth containing increasing concentrations of the antibiotic (4 to $512 \mu \mathrm{g} / \mathrm{ml}$ ) and $10 \mu \mathrm{l}$ of inoculum $\left(10^{5} \mathrm{CFU} / \mathrm{ml}\right.$, according to McFarland 0.5 solution) were added to each well. Negative controls, constituted of LB broth without antibiotic, were included in each test. The plates were incubated for $24 \mathrm{~h}$ at the optimal growth temperature of each bacterium, and the results were analyzed by visual inspection. The MIC corresponded to the concentration of the antibiotic that resulted in the absence of turbidity in the well. For confirmation of absence of viable cells, $2 \mu \mathrm{l}$ of the content of the well was inoculated on LB agar for growth visualization. The MBC value corresponded to the lowest concentration of kanamycin that prevented bacterial growth on the LB agar.

\section{Synergy Between Phenolic Compounds and Kanamycin}

The inhibitory effect of curcumin and resveratrol in combination with kanamycin on the growth of A. hydrophila IOC/FDA 110-36, S. Montevideo 163 and S. marcescens MG1 was evaluated 
by the checkerboard method, as described by Sanhueza et al. (2017). The tests were done in 96-well plates. The wells in rows contained phenolic compounds (resveratrol ranging from 25 to $100 \mu \mathrm{M}$ and curcumin ranging from 1.5 to $50 \mu \mathrm{M}$ ), and the wells in columns contained kanamycin (4 to $512 \mu \mathrm{g} / \mathrm{ml}$ ) plus LB broth, totaling $90 \mu \mathrm{l}$ in each well. Subsequently, $10 \mu \mathrm{l}$ of bacterial inoculum was added to each well $\left(10^{5} \mathrm{CFU} / \mathrm{ml}\right.$, according to McFarland 0.5 solution). The last row and column were used as controls, with only one agent and increasing concentrations of the second agent. The plates were incubated for $24 \mathrm{~h}$ at the optimal growth temperature of each bacterium, and growth curves were constructed based on $\mathrm{OD}_{595}$ measured every $2 \mathrm{~h}$ using a spectrophotometer (Multiskan FC, Thermo Fisher Scientific, Waltham, Massachussetts, United States).

\section{Statistical Analyses}

Experiments were performed in three replicates, and results were submitted to ANOVA followed by Tukey's test using the GraphPad Prism 8.0 software. A value of $p<0.05$ was considered as statistically significant.

\section{RESULTS}

\section{Selection of Phenolic Compounds by Molecular Docking}

Among the tested phenolic compounds, curcumin, capsaicin, resveratrol, and phloretin presented good molecular docking results, anchoring with good affinity to at least four of the six CviR structural variants. Based on their cost and commercial availability, they were selected for the in vitro studies. Gallic acid was also included due to its availability because it is a standard phenolic compound.

Figure 1 indicates how the tested phenolic compounds interacted with the 3QP1 protein. The interactions with the controls 3-oxo-C12-HSL (PubChem CID: 3246941) and Furanone C30 (PubChem CID: 10180544) are also shown. Ranking by the best binding affinities, 3-oxo-C12-HSL was in first place for structures 3QP1, 3QP4, and 3QP6, presenting GScores of $-83.91,-85.61$, and -89.6 , respectively. The lower GScores (more negative) correspond to better binding affinities (Almeida et al., 2016). The phenolic compounds presented weaker binding affinity than 3-oxo-C12-HSL for all protein structures. However, the negative GScores for resveratrol ( -57.30 to -66.62$)$, capsaicin ( -70.39 to -76.67$)$, curcumin $(-66.93$ to -75.82$)$, and phloretin ( -63.10 to -70.19$)$ indicated a good potential for binding to CviR QS regulator of C. violaceum (for the full list of GScores please see Supplementary Tables 2 and 3).

\section{Antibacterial Activity}

\section{Minimal Inhibitory Concentration}

Table 1 shows that gallic acid presented a MIC of $9.4 \mathrm{mM}$ over the two strains of C. violaceum (ATCC 12472 and 026). The MIC of the other compounds was higher than the highest tested concentration. Concentrations above this limit could not be tested due to issues related to the solubility of the compounds in the assay media.

\section{Anti-QS Activity of Phenolic Compounds Violacein Production}

The effect of the studied phenolic compounds on violacein production by C. violaceum ATCC 12472 is shown in Figure 2. The inhibition of pigment production by curcumin $(12 \mu \mathrm{M})$ and capsaicin $(70 \mu \mathrm{M})$ was $40 \%$, while resveratrol reached $60 \%$ inhibition, regardless of the tested concentration $(1.5,3.0$, or $6.0 \mu \mathrm{M})$, without affecting the growth of C. violaceum ATCC 12472. Gallic acid and phloridizin did not affect violacein production.

Figure 3 shows the effect of the phenolic compounds on violacein production by $C$. violaceum 026 in the presence of C6-HSL. C. violaceum 026 is not capable of producing the autoinducer C6-HSL due to a mutation in the cviI gene but maintains the ability to receive exogenous shortchain HSLs (4 to 6 carbons) as the $c v i R$ gene remains functional, resulting in production of the violacein pigment (Zhang et al., 2016). Among the evaluated phenolic compounds, only curcumin and capsaicin inhibited the production of violacein by this strain. The inhibition presented by curcumin was concentration-dependent, in which the highest tested concentration $(25 \mu \mathrm{M})$ inhibited $75 \%$ of pigment production.

As phloridizin and gallic acid did not affect violacein production, indicating that they did not present any anti-QS activity, they were excluded from the tests for motility and biofilm formation.

\section{Swarming and Swimming Motility}

Table 2 shows the effect of phenolic compounds on the swarming motility of $A$. hydrophila and $S$. marcescens. Salmonella Montevideo was unable to perform swarming motility in our study. Capsaicin and resveratrol did not inhibit the swarming motility of $A$. hydrophila $(p>0.05)$ at the evaluated concentrations, while curcumin induced the motility in this organism $(p<0.05)$. Conversely, curcumin effectively inhibited the swarming motility of $S$. marcescens, indicating the anti-QS potential of curcumin in this organism. In contrast, capsaicin and resveratrol did not inhibit this phenotype ( $p>0.05$; Supplementary Figure 1S).

Regarding swimming motility, none of the tested phenolic compounds inhibited this phenotype in A. hydrophila and $S$. marcescens ( $p>0.05$; Supplementary Figure $2 S)$.

\section{Biofilm Formation}

The effect of curcumin, capsaicin, and resveratrol on biofilm formation by $A$. hydrophila is shown in Figure 4. Curcumin (Figure 4A) and capsaicin (Figure 4B) did not inhibit this phenotype. In fact, capsaicin induced the biofilm formation in a concentration-dependent manner. In contrast, resveratrol (Figure 4C) significantly reduced the biofilm formation up to $80 \%$ at $100 \mu \mathrm{M}$.

Figure 5 shows the effect of curcumin, capsaicin, and resveratrol on biofilm formation by $S$. Montevideo. Inhibitory activity of curcumin (Figure 5A) and capsaicin (Figure 5B) 

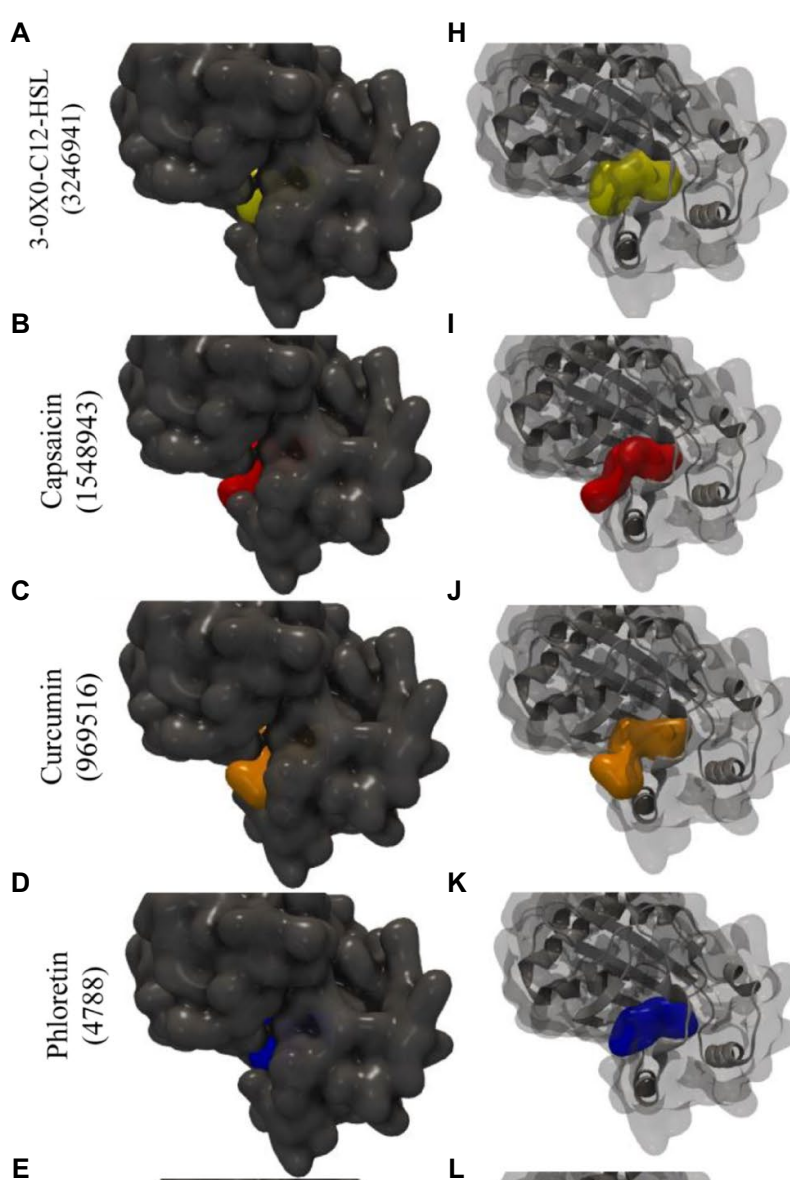

E
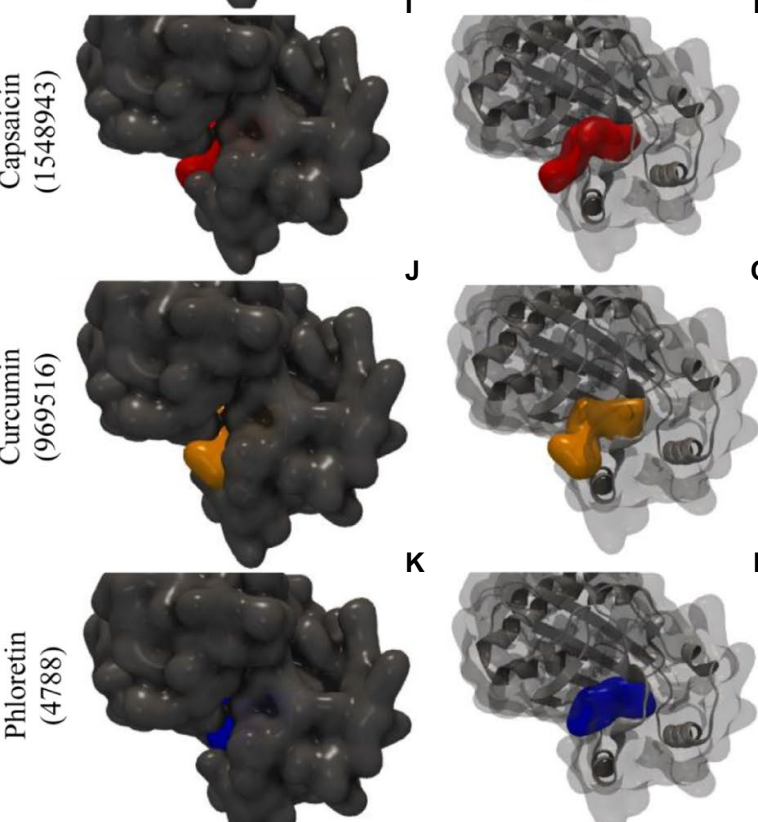

L
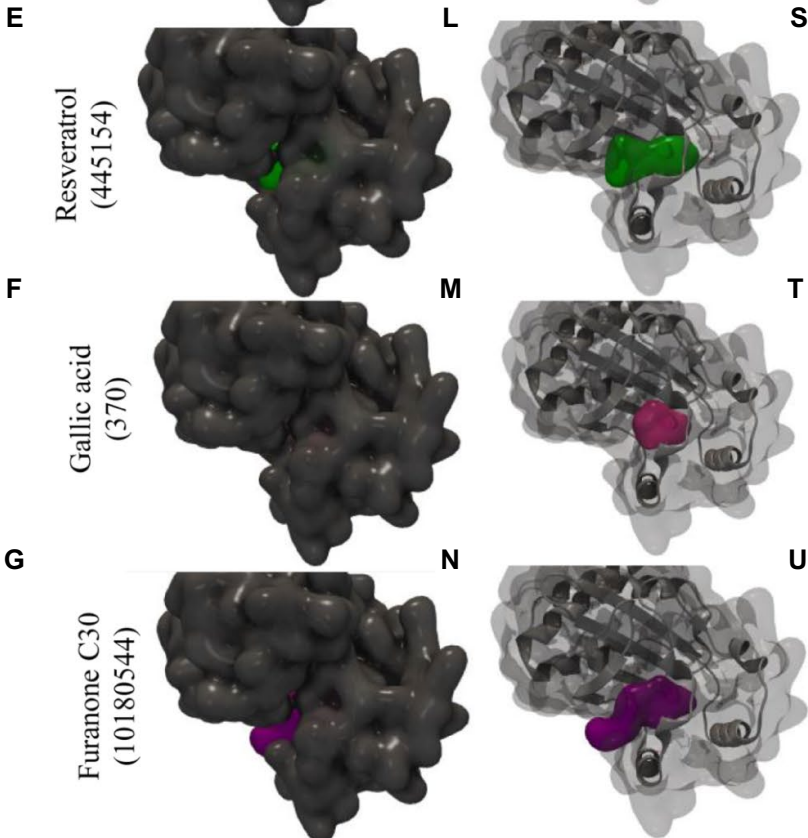

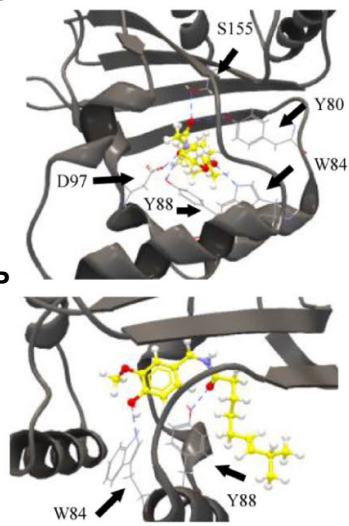

Q
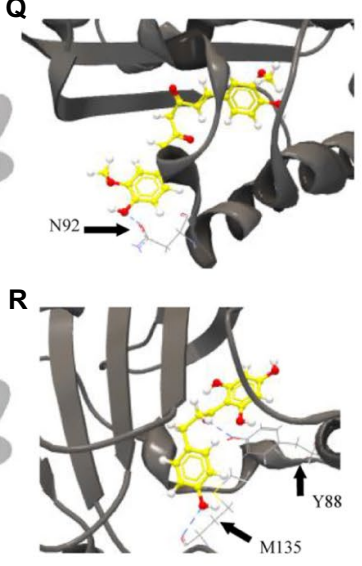

$\mathbf{S}$

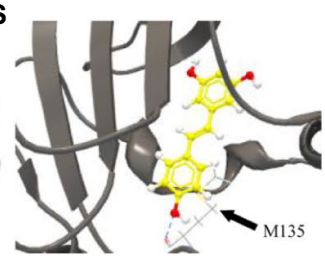

$\mathbf{T}$
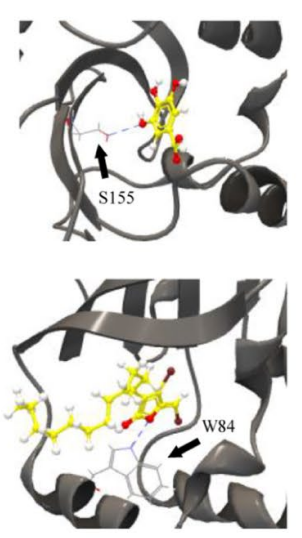

FIGURE 1 | Molecular docking of the 3QP1 protein of CviR QS regulator of Chromobacterium violaceum with 3-oxo-C12-HSL, capsaicin, curcumin, phloretin, resveratrol, gallic acid, and Furanone C30. Surface representation (A-G); surface and backbone representations (H-N); and backbone representations with hydrogen bonding between amino acid residues and phenolic compounds (0-U). Gray surface representation: CviR protein; yellow surface representation: 3-oxoC12-HSL; red surface representation: capsaicin; orange surface representation: curcumin; blue surface representation: phloretin; green surface representation: resveratrol; pink surface representation: gallic acid; and purple surface representation: Furanone C30. Black arrow indicates the binding site by hydrogen bonding. 
TABLE 1 | Minimum inhibitory concentration (MIC) of phenolic compounds.

\begin{tabular}{|c|c|c|c|c|c|}
\hline \multirow{2}{*}{ Target microorganism } & \multicolumn{5}{|c|}{ MIC (mM) } \\
\hline & Curcumin & Capsaicin & Phloridizin & Resveratrol & Gallic acid \\
\hline Aeromonas hydrophila IOC/FDA 110-36 & $>0.1$ & $>1.0$ & $>0.9$ & $>0.1$ & $>9.4$ \\
\hline Chromobacterium violaceum ATCC12472 & $>0.1$ & $>1.0$ & $>0.9$ & $>0.1$ & 9.4 \\
\hline Chromobacterium violaceum $\mathrm{O} 26$ & $>0.1$ & $>1.0$ & $>0.9$ & $>0.1$ & 9.4 \\
\hline Salmonella Montevideo 163 & $>0.1$ & $>1.0$ & $>0.9$ & $>0.1$ & $>9.4$ \\
\hline Serratia marcescens MG1 & $>0.1$ & $>1.0$ & $>0.9$ & $>0.1$ & $>9.4$ \\
\hline
\end{tabular}

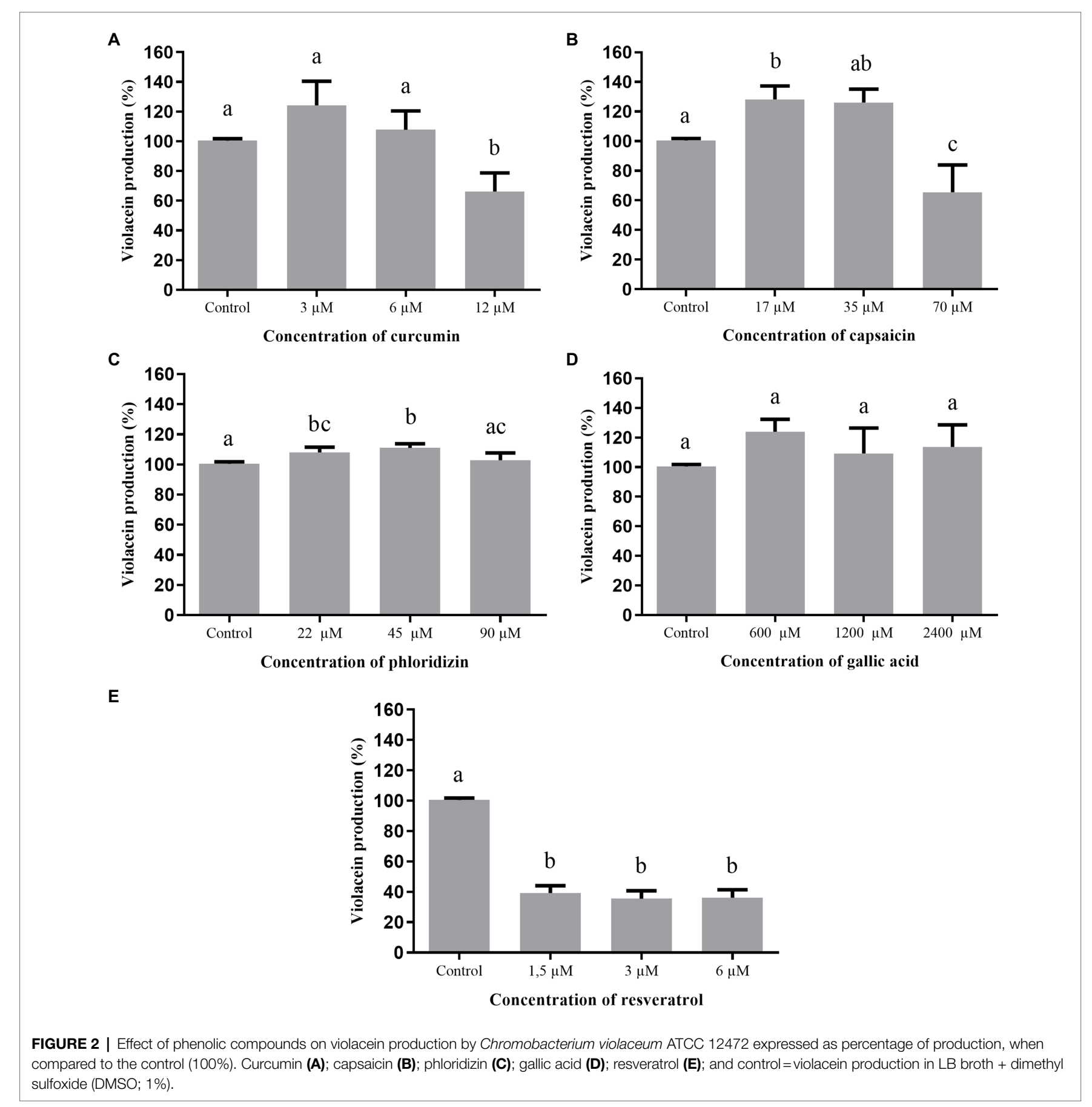




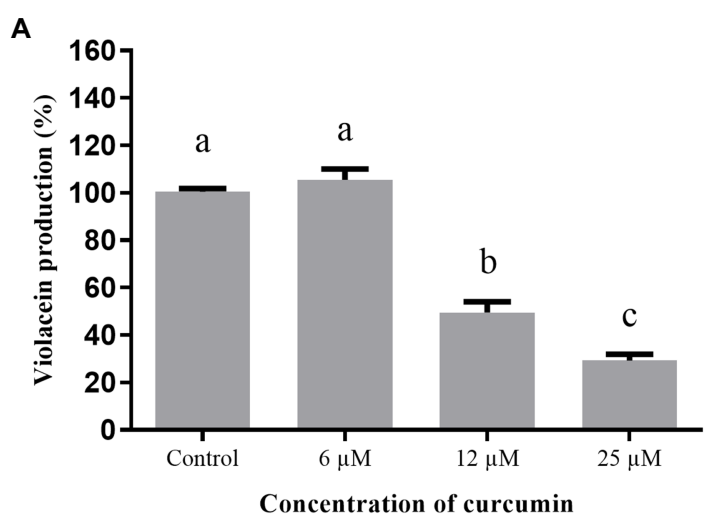

C

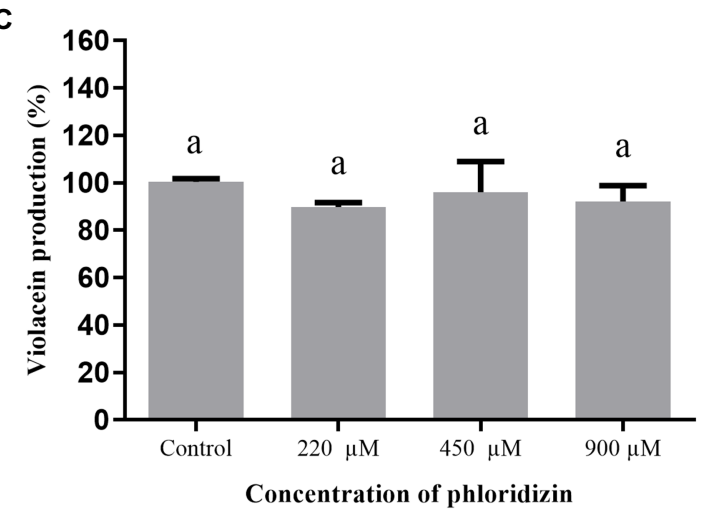

B
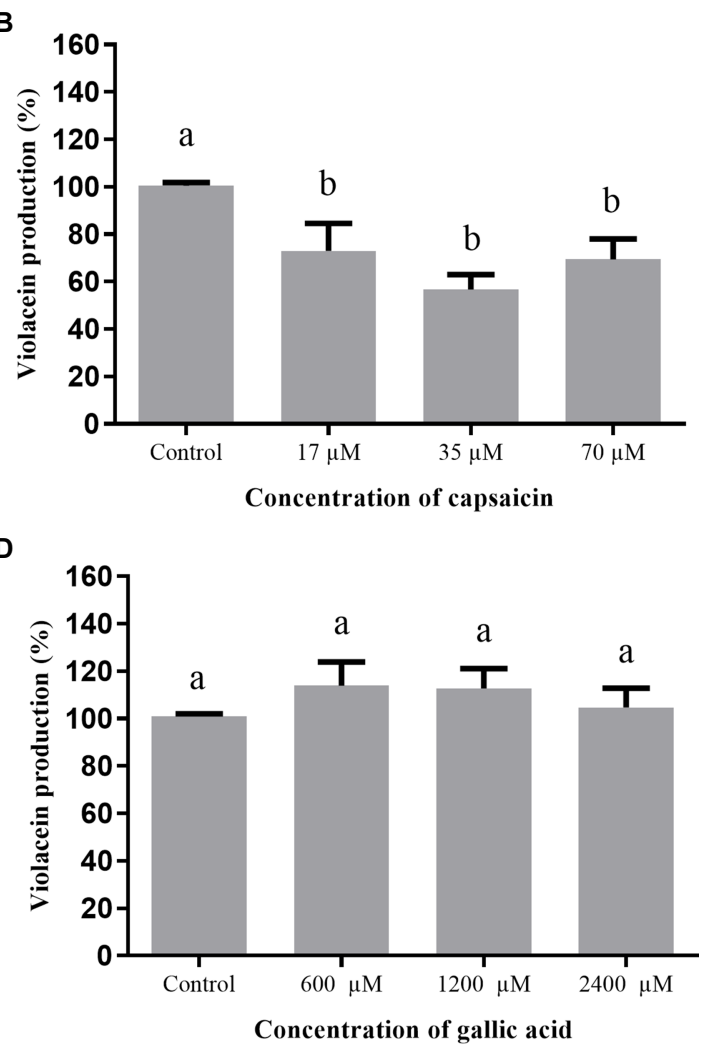

E

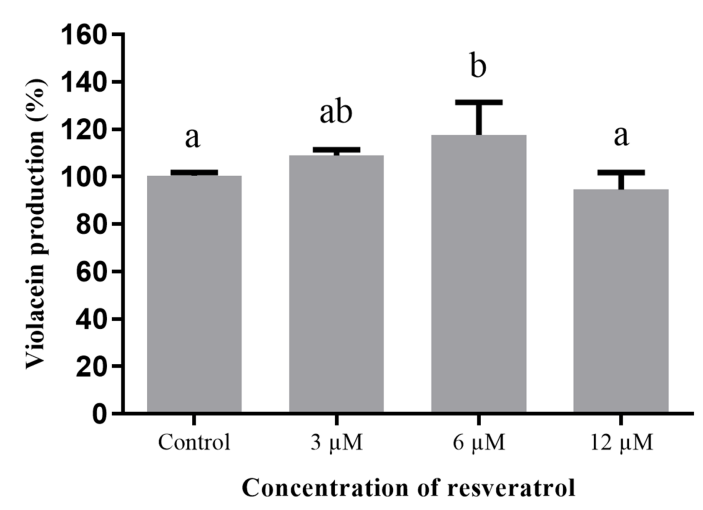

FIGURE 3 | Effect of phenolic compounds on violacein production by Chromobacterium violaceum 026 in the presence of C6-HSL, expressed as percentage of production, when compared to the control (100\%). curcumin (A); capsaicin (B); phloridizin (C); gallic acid (D); resveratrol (E); and control=violacein production in LB broth + DMSO (1\%).

was similar; i.e., both were able to inhibit biofilm formation up to $27 \%$ when compared to the control $(p<0.05)$. In contrast, resveratrol (Figure 5C) did not inhibit biofilm formation at the tested concentrations $(p>0.05)$.

When the phenolic compounds were tested for inhibition of biofilm formation by S. marcescens (Figure 6), curcumin (Figure 6A) was ineffective $(p>0.05)$. Capsaicin (Figure 6B) and resveratrol (Figure 6C) were active at the highest tested concentrations; i.e., $1,000 \mu \mathrm{M}$ capsaicin and $100 \mu \mathrm{M}$ resveratrol were able to inhibit biofilm formation up to $43 \%$.

\section{Effect of Phenolic Compounds (Curcumin and Resveratrol) Combined With Kanamycin on Growth}

Figure 7 presents the growth curves of A. hydrophila, $S$. Montevideo, and $S$. marcescens in LB broth containing curcumin or resveratrol in combination with kanamycin, at varied concentrations. The curves clearly indicate that the two phenolic compounds delayed the growth of all tested bacteria when combined with sub-inhibitory concentrations of the antibiotic, in a concentration-dependent pattern. 
TABLE 2 | Effect of capsaicin, curcumin, and resveratrol on swarming motility of Aeromonas hydrophila IOC/FDA $110-36$ and Serratia marcescens MG1.

\begin{tabular}{|c|c|c|c|c|}
\hline \multirow{2}{*}{ Phenolic compound } & \multicolumn{4}{|c|}{ Radius of swarming zone after $12 \mathrm{~h}(\mathrm{~mm})$} \\
\hline & Tested concentration & Aeromonas hydrophila & Tested Concentration & Serratia marcescens \\
\hline \multirow[t]{4}{*}{ Capsaicin } & $0 \mu \mathrm{M}$ & $12.0 \pm 0.0^{a}$ & $0 \mu \mathrm{M}$ & $20.0 \pm 0.0^{\mathrm{a}}$ \\
\hline & $65 \mu \mathrm{M}$ & $12.2 \pm 0.3^{a}$ & $65 \mu \mathrm{M}$ & $30.0 \pm 1.4^{b}$ \\
\hline & $130 \mu \mathrm{M}$ & $9.5 \pm 2.1^{\mathrm{a}}$ & $130 \mu \mathrm{M}$ & $31.5 \pm 0.7^{b}$ \\
\hline & $260 \mu \mathrm{M}$ & $8.7 \pm 1.0^{\mathrm{a}}$ & $260 \mu \mathrm{M}$ & $32.5 \pm 0.7^{b}$ \\
\hline \multirow[t]{4}{*}{ Curcumin } & $0 \mu \mathrm{M}$ & $12.5 \pm 0.7^{\mathrm{ab}}$ & $0 \mu \mathrm{M}$ & $49.0 \pm 0.0^{\mathrm{a}}$ \\
\hline & $3 \mu \mathrm{M}$ & $15.2 \pm 1.0^{\mathrm{abc}}$ & $12.5 \mu \mathrm{M}$ & $5.5 \pm 0.7^{b}$ \\
\hline & $6 \mu \mathrm{M}$ & $16.5 \pm 0.0^{\mathrm{bc}}$ & $25 \mu \mathrm{M}$ & $5.0 \pm 0.0^{b}$ \\
\hline & $12 \mu \mathrm{M}$ & $18.7 \pm 3.1^{c}$ & $50 \mu \mathrm{M}$ & $5.0 \pm 1.4^{b}$ \\
\hline \multirow[t]{4}{*}{ Resveratrol } & $0 \mu \mathrm{M}$ & $13.5 \pm 2.1^{\mathrm{a}}$ & $0 \mu \mathrm{M}$ & $22.5 \pm 2.1^{\mathrm{ab}}$ \\
\hline & $25 \mu \mathrm{M}$ & $14.0 \pm 0.0^{a}$ & $25 \mu \mathrm{M}$ & $31.0 \pm 1.4^{b}$ \\
\hline & $50 \mu \mathrm{M}$ & $13.7 \pm 0.3^{a}$ & $50 \mu \mathrm{M}$ & $27.0 \pm 2.8^{b}$ \\
\hline & $100 \mu \mathrm{M}$ & $14.0 \pm 0.0^{a}$ & $100 \mu \mathrm{M}$ & $21.0 \pm 0.0^{\mathrm{ab}}$ \\
\hline
\end{tabular}

Results expressed as mean \pm standard deviation. Means followed by different superscript letters $(a, b, c)$ in the same column differ among themselves by Tukey's test ( $p<0.05)$.

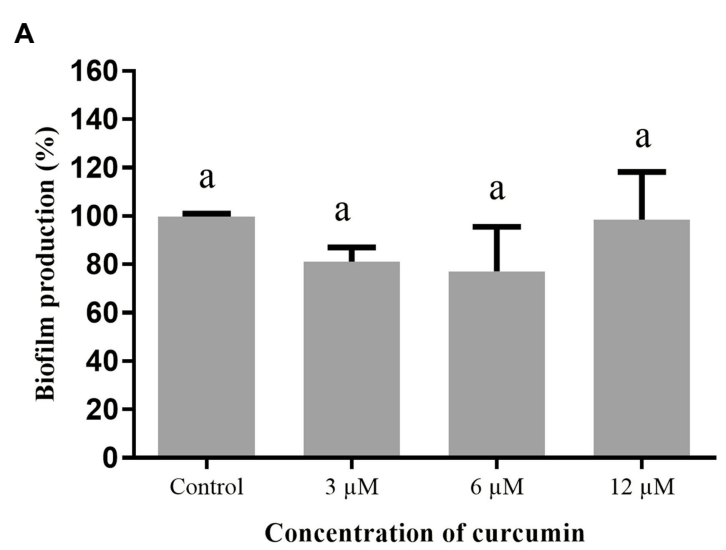

C

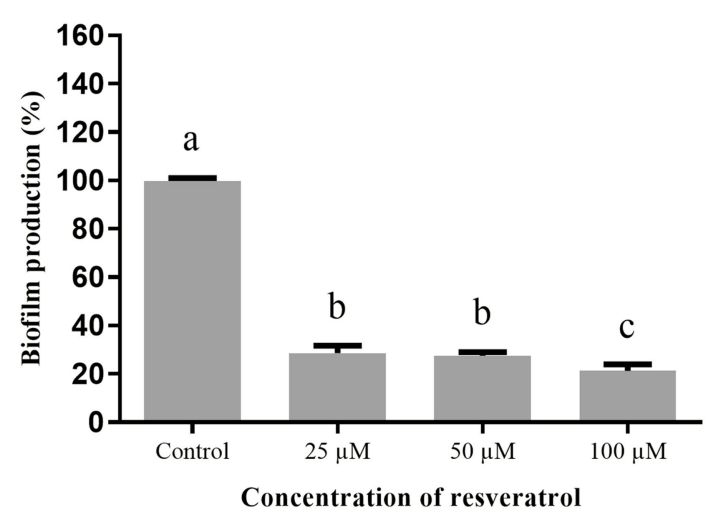

FIGURE 4 | Effect of phenolic compounds on biofilm formation by Aeromonas hydrophila IOC/FDA 110-36, expressed as percentage of production, when compared to the control (100\%). curcumin (A); capsaicin (B); resveratrol (C); and control=biofilm formation in LB broth + DMSO (1\%). Means followed by different letters differ among themselves by Tukey's test $(p<0.05)$.

For A. hydrophila, $256 \mu \mathrm{g} / \mathrm{ml}$ of kanamycin held the bacterial growth for at least $14 \mathrm{~h}$. Growth in the presence of curcumin $(3-12 \mu \mathrm{M})$ was slowed for longer periods of time (Figure 7A). Lower tested concentrations of kanamycin $(4-128 \mu \mathrm{g} / \mathrm{ml})$ did not affect the growth of this bacterium (Supplementary Figure 3S). When the concentration of

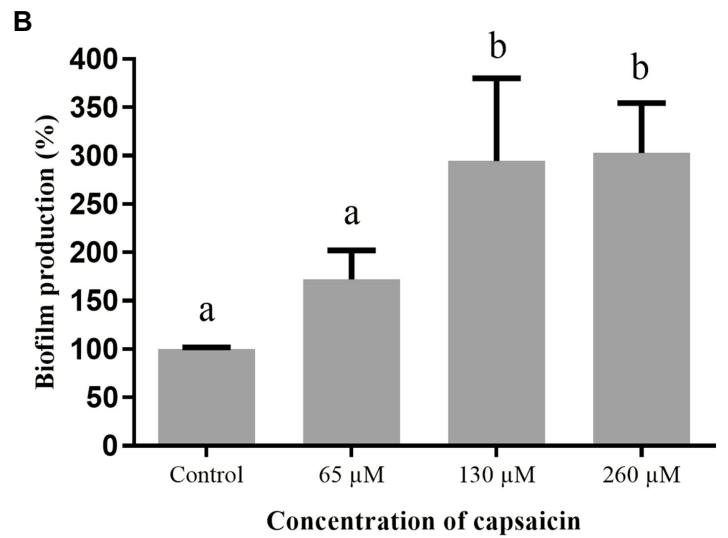



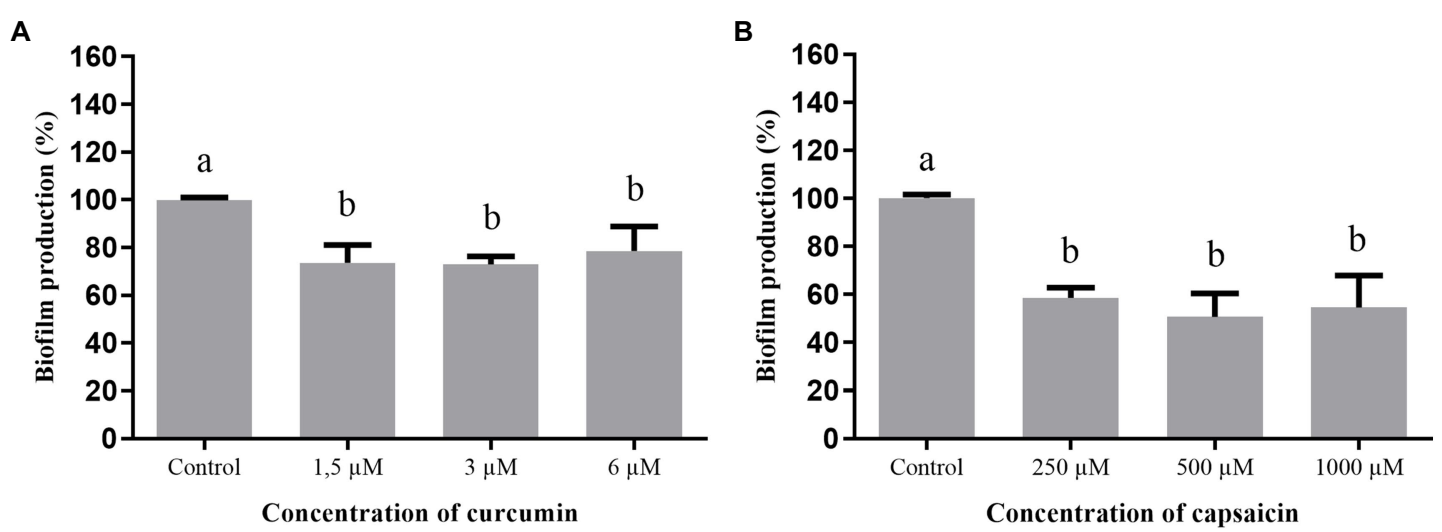

C

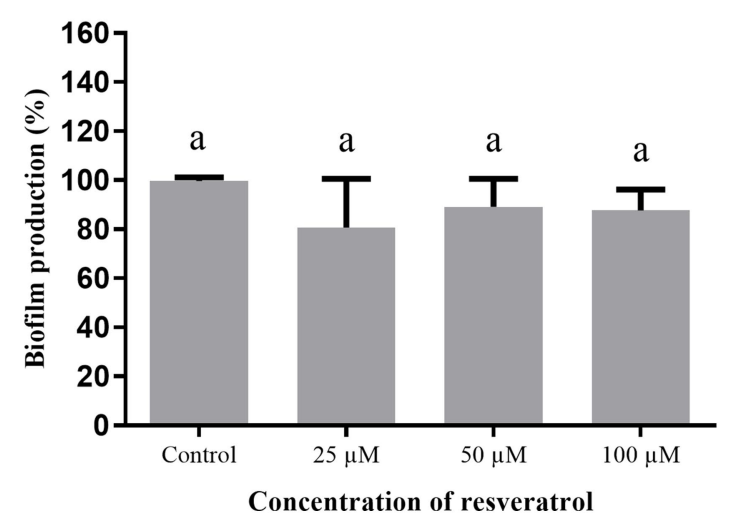

FIGURE 5 | Effect of phenolic compounds on biofilm formation by Salmonella Montevideo 163 expressed as percentage of production, when compared to the control (100\%). curcumin (A); capsaicin (B); resveratrol (C); and control = biofilm formation in LB broth + DMSO (1\%). Means followed by different letters differ among themselves by Tukey's test $(p<0.05)$.

(Supplementary Figure 4S). Higher concentrations of kanamycin (64 to $512 \mu \mathrm{g} / \mathrm{ml}$ ) caused the complete inhibition of bacterial growth (Supplementary Figure 4S).

For $S$. marcescens, the combination of curcumin with kanamycin in inhibiting growth was less effective than for A. hydrophila and S. Montevideo (Figure 7C). Evidence of inhibition was observed only when $32 \mu \mathrm{g} / \mathrm{ml}$ of kanamycin was combined with curcumin, being slightly more effective at $50 \mu \mathrm{M}$ of the later. Lower concentrations of kanamycin (4 to $16 \mu \mathrm{g} / \mathrm{ml}$ ) and curcumin $(0-50 \mu \mathrm{M})$ resulted in no reduction in the microbial growth, while higher concentrations of kanamycin (64 to $512 \mu \mathrm{g} / \mathrm{ml}$ ) resulted in stronger growth inhibition that was not affected by the presence of curcumin (Supplementary Figure 5S).

Results of the tests with resveratrol $(0-100 \mu \mathrm{M})$ combined with kanamycin $(4-512 \mu \mathrm{g} / \mathrm{ml})$ are shown in Figure $7 D-F$. The growth curves of A. hydrophila (Figure 7D), S. Montevideo (Figure 7E), and $S$. marcescens (Figure 7E) indicate that the effects of these combinations were similar to those observed for the curcumin-kanamycin combinations.

The curves from assays with kanamycin (4 to $64 \mu \mathrm{g} / \mathrm{ml}$ ) and resveratrol $(0-100 \mu \mathrm{M})$ show partial inhibition of growth of $A$. hydrophila from 8 to $10 \mathrm{~h}$, compared with the curve from assays using only kanamycin and the control (Supplementary Figure 6S). Kanamycin at $128 \mu \mathrm{g} / \mathrm{ml}$ combined with resveratrol presented a growth delay, in which the bacterial growth started at $14 \mathrm{~h}$ of incubation, while with the treatment containing only kanamycin the growth started at $8 \mathrm{~h}$ of incubation (Figure 7D). The results show that resveratrol made A. hydrophila more sensitive to kanamycin at the studied concentrations (Supplementary Figure 6S).

The lowest concentrations of kanamycin (4 and $8 \mu \mathrm{g} / \mathrm{ml}$ ) did not inhibit the growth of $S$. Montevideo. In the concentration of $32 \mu \mathrm{g} / \mathrm{ml}$ of kanamycin, there was growth retardation in all concentrations of resveratrol evaluated, especially at $100 \mu \mathrm{M}$, with a delay of $6 \mathrm{~h}$ compared to the other treatments (Figure 7E). In the highest concentrations of antibiotic, there was no microbial growth; thus, $64 \mu \mathrm{g} / \mathrm{ml}$ is the $\mathrm{MBC}$ of this microorganism (Supplementary Figure 7S).

In the test with $S$. marcescens, at concentrations from 4 to $16 \mu \mathrm{g} / \mathrm{ml}$ of kanamycin, growth in the presence of resveratrol was strongly inhibited. The highest concentration of resveratrol $(100 \mu \mathrm{M})$ reduced the MIC 3-fold when compared to the curve containing only kanamycin (Figure $7 \mathbf{F}$ ). At concentrations of 32 to $128 \mu \mathrm{g} / \mathrm{ml}$ of kanamycin, resveratrol completely inhibited growth. It was observed that resveratrol made the microorganism more sensitive to the antibiotic. Finally, complete inhibition of growth was observed in the presence of 256 and $512 \mu \mathrm{g} / \mathrm{ml}$ kanamycin alone (Supplementary Figure 8S). 
A

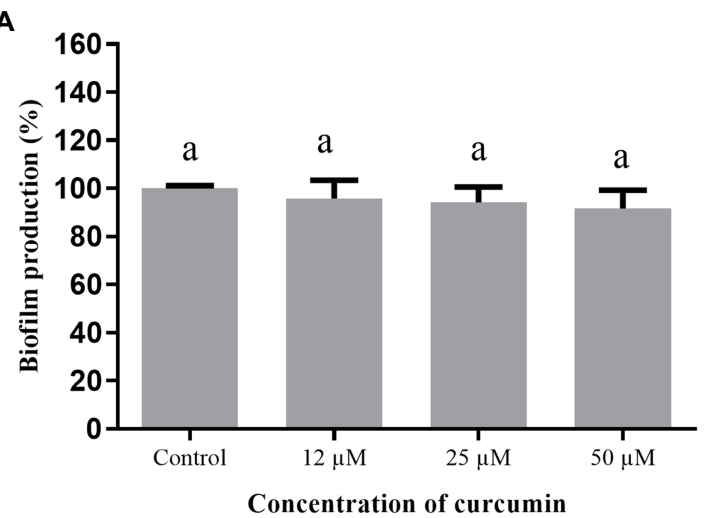

B

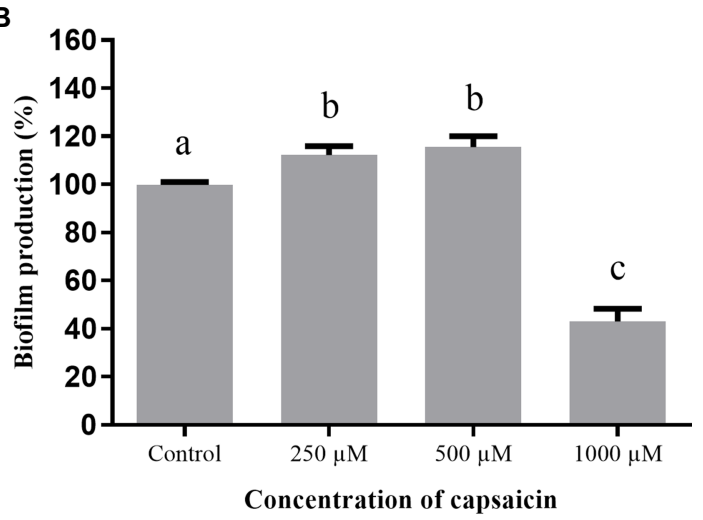

C

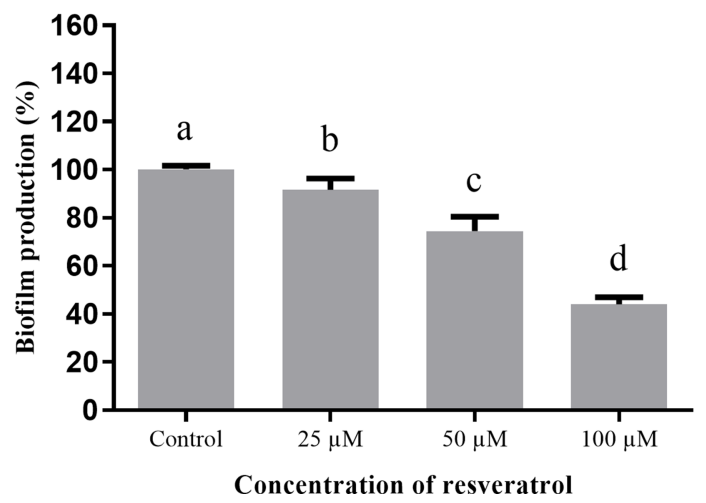

FIGURE 6 | Effect of phenolic compounds on biofilm formation by Serratia marcescens MG1 expressed as percentage of production, when compared to the control (100\%). Curcumin (A); capsaicin (B); resveratrol (C); and control=biofilm formation in LB broth + DMSO (1\%). Means followed by different letters differ among themselves by Tukey's test $(p<0.05)$.

A

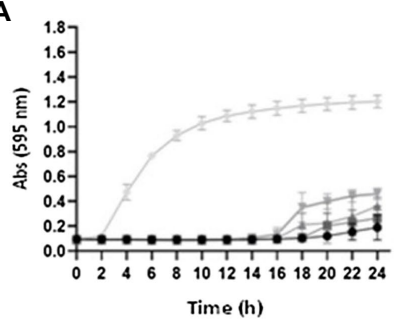

D

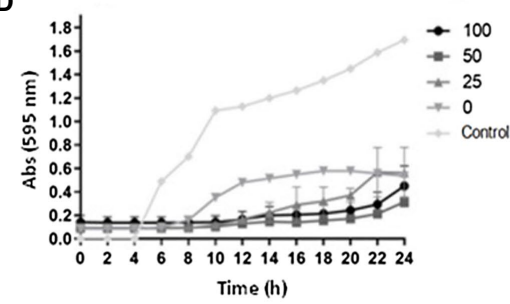

B

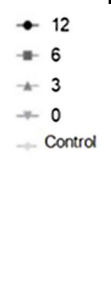

E

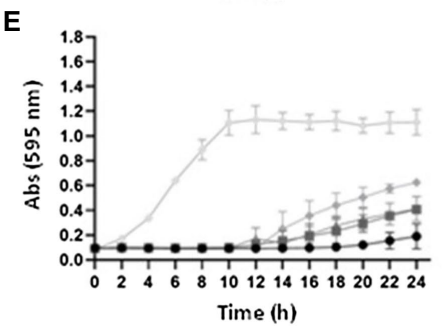

C
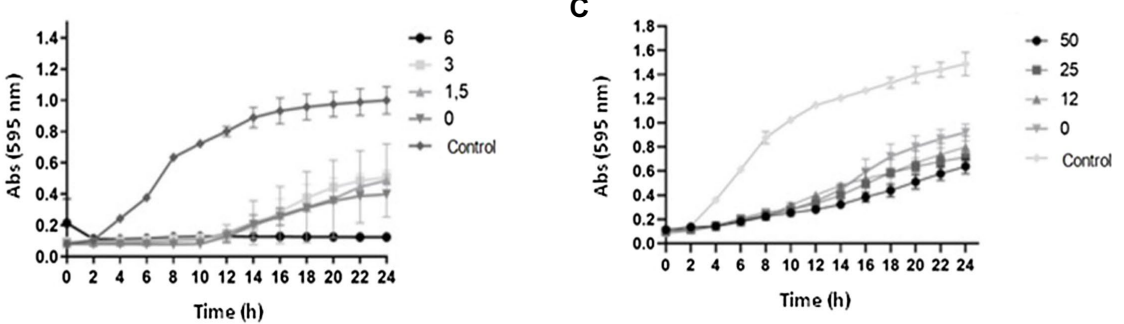

$\mathbf{F}$
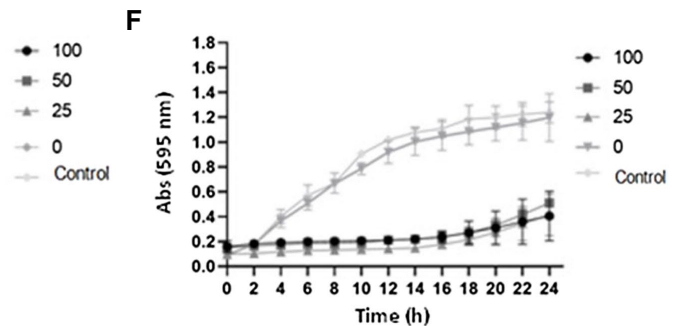

FIGURE 7 | Growth curves of bacteria treated with phenolic compounds and kanamycin. (A) A. hydrophila IOC/FDA 110-36 treated with curcumin (0, 3, 6, and $12 \mu \mathrm{M})$ and kanamycin $(256 \mu \mathrm{g} / \mathrm{ml})$. (B) Salmonella Montevideo 163 treated with curcumin $(0,1.5,3$, and $6 \mu \mathrm{M})$ and kanamycin $(32 \mu \mathrm{g} / \mathrm{ml})$. (C) Serratia marcescens MG1 treated with curcumin $(0,12,25$, and $50 \mu \mathrm{M})$ and kanamycin $(32 \mu \mathrm{g} / \mathrm{ml})$. (D) A. hydrophila IOC/FDA 110-36 treated with resveratrol $(0,25,50$, and 100 $\mu \mathrm{M})$ and kanamycin $(128 \mu \mathrm{g} / \mathrm{ml})$. (E) Salmonella Montevideo treated with resveratrol $(0,25,50$, and $100 \mu \mathrm{M})$ and kanamycin $(32 \mu \mathrm{g} / \mathrm{ml})$. (F) Serratia marcescens treated with resveratrol $(0,25,50$, and $100 \mu \mathrm{M})$ and kanamycin $(4 \mu \mathrm{g} / \mathrm{ml})$. Control=bacterial growth in LB broth + DMSO $(1 \%)$. 


\section{DISCUSSION}

Phenolic compounds are known for their antimicrobial effects as a consequence of structural or functional damages to the bacterial cell membrane (Silva et al., 2018). Besides, phenolic acids can affect the physicochemical properties of the bacterial cell surface, especially hydrophobicity, in addition to altering electron receptors and polar and non-polar components of bacteria (Borges et al., 2013). Furthermore, phytochemicals, such as phenolic compounds, may compete with AHL signaling molecules for binding to QS receptors (Nazzaro et al., 2013; Almeida et al., 2018; Aswathanarayan and Vittal, 2018; Quecán et al., 2018; Santos et al., 2020), suggesting possible interactions of these compounds with LuxR-type proteins, like the CviR QS regulator of Chromobacterium violaceum (Figure 1).

Curcumin, one of the selected phenolic compounds for the in vitro studies, is the main polyphenol in the rhizome of Curcuma longa. Beyond its numerous pharmaceutical properties, such as antitumor, anti-HIV-1, and antibacterial activity, this compound also presents anti-QS activity, inhibiting several phenotypes in Pseudomonas aeruginosa PAO1, Escherichia coli, Serratia marcescens, and Proteus mirabilis (Ding et al., 2017). Resveratrol is also active against various pathogens and presents anti-QS activity, being capable of inhibiting biofilm formation and dispersing established biofilms (Duarte et al., 2015). Gallic acid is a phenolic acid present in numerous foods, and also an antimicrobial agent, affecting bacterial cell membranes and causing irreversible changes in permeability, rupture, and pore formation (Sorrentino et al., 2018). Capsaicin and luteolin are polyphenols abundant in several pepper species, especially those of the Capsicum genus, and have the ability to inhibit microbial growth, in addition to antioxidant, anti-inflammatory, cardioprotective, neuroprotective, and anticancer activity (Basith et al., 2016; Rivera et al., 2019).

The antimicrobial activity of phloridizin in the tested concentrations against the target microorganisms was lower when compared to the other phenolic compounds. Barreca et al. (2014) evaluated the antimicrobial activity of phloridizin and phloretin, its aglycone form, against several Gram-positive and Gram-negative bacteria, and observed that the presence of glucose in the basic chalcone structure caused a reduction in antimicrobial activity. Furthermore, they also observed that phloretin is particularly active against Gram-positive bacteria. The low activity against the tested Gram-negative microorganisms observed in this study corroborate the findings of Barreca et al. (2014). Interestingly, Quecán et al. (2019) also observed that glycosylation of quercetin reduces its antimicrobial and anti-QS effect.

Regarding the determination of the MIC of phenolic compounds, it is crucial to perform QS assays in concentrations that do not interfere with bacterial growth, since this would affect quantification of QS regulated phenotypes due to cell density differences (Defoirdt et al., 2013; Maura et al., 2016). Bali et al. (2019) evaluated the MIC of curcumin $(1,500 \mu \mathrm{g} / \mathrm{ml}$, equivalent at $4.1 \mathrm{mM})$ and gallic acid $(>1,500 \mu \mathrm{g} / \mathrm{ml}$, equivalent to $>8.8 \mathrm{mM}$ ) in C. violaceum ATCC 12472 , corroborating the results of the present work (Table 1). Packiavathy et al. (2014) also evaluated anti-QS activity of curcumin and reported an MIC of $384 \mu \mathrm{g} / \mathrm{ml}$, equivalent to $1.04 \mathrm{mM}$, for C. violaceum 026, a value higher than that tested in the present study (0.1 mM). Rivera et al. (2019) studied compounds present in Capsicum frutescens pepper extract and found that MIC of capsaicin was higher than $100 \mu \mathrm{g} / \mathrm{ml}$ (equivalent to $0.34 \mathrm{mM}$ ). The growth of $C$. violaceum ATCC 12472 and 026 was partially inhibited by the compound at $0.1 \mathrm{mM}$, which is similar to the results found in the present work.

Curcumin inhibited violacein production by $C$. violaceum ATCC 12472 (Figure 2), as also observed by Bali et al. (2019). However, those authors evaluated higher concentrations $(2 \mathrm{mM}$, $1.02 \mathrm{mM}$, and $0.51 \mathrm{mM}$ ) than those used in the present work $(0.012 \mathrm{mM}, 0.006 \mathrm{mM}$ and $0.003 \mathrm{mM})$, indicating that even at lower concentrations, curcumin consistently inhibits this QS regulated phenotype. It should be noted that in concentrations higher than $0.050 \mathrm{mM}$, a partial inhibition of microbial growth was observed in our study (results not shown), which should affect violacein production in a QS-independent manner.

In the study of Rivera et al. (2019), capsaicin did not inhibit violacein production when the concentration was similar to the one used in this study. However, those authors did not test the concentration of $70 \mu \mathrm{M}$, which was the one with best anti-QS activity detected here. When working with resveratrol, Duarte et al. (2015) found positive results for violacein inhibition, corroborating the results of this work and the potential of this compound. They further suggested that the anti-QS activity of resveratrol is due to its ability to mimic QS signals and disrupt bacterial communication (Duarte et al., 2015). In the present study, phloridizin and gallic acid did not show anti-QS activity in C. violaceum ATCC 12472. Borges et al. (2014) and Bali et al. (2019) also reported that gallic acid did not exhibit anti-QS activity in the biosensor strain.

Tests performed with only wild-type $C$. violaceum strain are not enough to detect the QS inhibitory mechanism, since the inhibition of violacein production may be due to reduced production of the autoinducer (inhibition of AHL synthesis by CviI) or by interference with the AHL-dependent transcriptional activator, the CviR protein (Rivera et al., 2019). Thus, besides C. violaceum ATCC 12472, complementary assays performed with additional strains, such as C. violaceum 026, a mutant unable to produce AHLs but still capable to respond to exogenous AHLs, can be informative (Rivera et al., 2019). If the tested compounds interfere with C6-HSL detection by CviR, a reduced violacein production by C. violaceum 026 can be expected (LaSarre and Federle, 2013). Thus, curcumin and capsaicin likely present anti-QS activity via AHL detection by CviR since these compounds reduced violacein production in C. violaceum 026 (Figure 3). Brackman et al. (2009) and Packiavathy et al. (2014) also observed inhibition of violacein production by curcumin in C. violaceum 026, even though they have used higher concentrations than those used in this study.

Swarming motility is an organized microbial movement on surfaces, dependent on extensive flagellation, cell-cell contact, and QS. This type of motility is associated with virulence and antibiotic resistance of various microorganisms, and is considered 
a favorable adaptation to the challenges that arise in dynamic environments, contributing to biofilm formation and infection (Rütschlin and Böttcher, 2019; Carette et al., 2020). Interference with this phenotype is an important alternative to reduce or prevent biofilm-based infections. In this study, curcumin effectively inhibited swarming motility (Table 2 and Supplementary Figure S1), which corroborates results reported by Packiavathy et al. (2014), who observed the inhibition of swarming motility of $S$. marcescens, E. coli, P. aeruginosa, and P. mirabilis by curcumin at $100 \mu \mathrm{g} / \mathrm{ml}(135 \mu \mathrm{M})$. One explanation for the observed inhibition is the ability of curcumin in reducing the production of extracellular polysaccharides by some pathogens, negatively affecting their motility (Zheng et al., 2020).

Swimming-type inhibition by curcumin liposomes was also observed for A. hydrophila and Serratia grimesii (Ding et al., 2018) and for S. marcescens, E. coli, P. aeruginosa, and P. mirabilis (Packiavathy et al., 2014). Other studies also observed motility inhibition by phenolic compounds, like proanthocyanidins and tannins that completely inhibited swarming, but did not block swimming (O'May and Tufenkji, 2011), and trans-resveratrol, that had greater activity on swarming than on swimming motility, indicating that these movement phenotypes have different activation mechanisms.

Regarding the inhibition of biofilm formation by A. hydrophila (Figure 4), only resveratrol showed a significant effect. Similar results were found in other studies using different bacteria: L. monocytogenes (Vasquez-Armenta et al., 2018); Campylobacter spp. and Arcobacter butzleri (Duarte et al., 2015); methicillin-resistant S. aureus (MRSA; Qin et al., 2014); and E. coli O157: H7 (Lee et al., 2013), evidencing the broad inhibitory effect of resveratrol.

Mangoudehi et al. (2020) evaluated curcumin at different concentrations against biofilm formation of $A$. hydrophila ATCC 7966 and other three strains isolated from fish (silver carps) and found $67 \%$ reduction of biofilm formation at $43.4 \mu \mathrm{M}$. These results differ from those observed in the present study (Figure 4A), where the maximum concentration was $12 \mu \mathrm{M}$, indicating that biofilm inhibition is concentration-dependent. On the other hand, curcumin inhibited the biofilm formation by $S$. Montevideo (Figure 5). The efficacy of curcumin against various infectious organisms results from multiple mechanisms, including its ability to disrupt bacterial membranes, inhibit replication, and alter gene expression (Vaughn et al., 2017). Packiavathy et al. (2014) observed a visible reduction in microcolonies, disruption of biofilm architecture, and reduced biofilm biomass of $P$. aeruginosa, S. marcescens, E. coli, and $P$. mirabilis treated with curcumin.

Biofilm formation by $S$. Montevideo was also inhibited by capsaicin (Figure 5). This compound is used as a food additive and flavoring, and has important pharmacological actions, such as bacteriostatic activity against Gram-negative bacteria and anti-inflammatory effects (Zhou et al., 2014). Some studies have shown that capsaicin inhibits adhesion, growth, and biofilm formation of Gram-negative bacteria, such as Pseudomonas putida, Vibrio matriegens, Vibrio parahaemolyticus (Xu et al., 2005), and Porphyromonas gingivalis (Zhou et al., 2014).

Regarding the inhibition of biofilm formation of $S$. marcescens (Figure 6) by capsaicin at $1,000 \mu \mathrm{M}$ and resveratrol at $25 \mu \mathrm{M}$ or higher, interesting effects were observed. The capability of trans-resveratrol to inhibit biofilm formation by enterohemorrhagic E. coli O157:H7 ATCC43895 (Lee et al., 2013), P. aeruginosa PAO1 and PA14 (Cho et al., 2013), and S. aureus (Morán et al., 2014) has already been described. Thus, resveratrol can be considered a potential anti-biofilm compound for several biofilm-forming microorganisms and its use should be further investigated on food-contacting materials.

Antimicrobial resistance is a serious concern in clinical practice. One strategy that is useful in combating multidrugresistant bacteria is the coadministration of antibiotics with adjuvants that modify bacterial resistance. Several medicines of great utility have been approved using this concept, such as the use of amoxicillin with beta-lactamase inhibitors. Other studies also show the effective combination of antibiotics with efflux-pump inhibitors (Ayaz et al., 2019). As biofilm formation is associated with reduced sensitivity to antibiotics, and it is known that QS regulates the expression of some genes associated with this phenotype, QS interference can be explored as an alternative to reduce bacterial virulence and increase the efficacy of antibiotic treatment (Mion et al., 2019). This study showed that curcumin at $6 \mu \mathrm{M}$ reduced the MIC of kanamycin by half in S. Montevideo (Figure 7B). These results corroborate those of Packiavathy et al. (2014) who observed increased sensitivity of $S$. marcencens to different antibiotics when combined with curcumin. Mun et al. (2013) and Kali et al. (2016) also observed a decrease in the MIC of several antibiotics against MRSA and other bacteria, when applied in combination with curcumin. These studies demonstrate that this phenolic compound can increase the sensitivity of several pathogens to antibioticos and is a potential agent to be coadministered with these drugs.

Serratia marcescens may be resistant to several antibiotics, including beta-lactam, aminoglycosides, and fluoroquinolones, hindering the treatment against infections caused by this bacterium (Yang et al., 2012). Like other Enterobacteriaceae, the production of beta-lactamase enzyme which inactivate beta-lactam antibiotics is the most common resistance mechanism in this micro-organism (Yang et al., 2012). The increased sensitivity of $S$. marcescens to colistin when combined with resveratrol reported by Cannatelli et al. (2018), the potentiation of the effects of aminoglycosides against biofilms of $P$. aeruginosa PAO1 (Zhou et al., 2018), and the increased sensitivity of multidrug resistant Klebsiella pneumoniae and E. coli (Liu et al., 2020b) and S. aureus (Liu et al., 2020a) deserve to be better explored as effective therapeutic alternatives.

The combination of bioactive compounds with antimicrobials seems to be a promising approach to improve the efficacy of these drugs. However, there are several challenges to be faced before translating these in vitro findings into real-life applications. Some of the challenges we envision are related to interactions and metabolization of these compounds in the human body, which could hinder their antimicrobial and anti-quorum sensing activities in vivo. Additionally, as pointed out by Vipin et al. (2020) and observed in the present work, strain variation in response to bioactive compounds is another challenge in treating infections. 


\section{CONCLUSION}

Phenolic compounds have potential to inhibit quorum sensing in foodborne bacteria. In silico analyses showed that gallic acid, capsaicin, curcumin, phloridizin, and resveratrol could interact with CviR QS receptor of C. violaceum. Curcumin and resveratrol presented better activity against violacein production, swarming motility, biofilm formation, and concomitant use with an antibiotic for increased microbial sensitivity. The present study and several others from the literature indicate that curcumin and resveratrol are potential QSI and have antimicrobial properties that encourage studies that could translate these findings to applications in the food and pharmaceutical industries. In order to characterize the specificity of quorum sensing inhibition, biochemical and genetic studies should be performed with these compounds and model organisms.

\section{DATA AVAILABILITY STATEMENT}

The raw data supporting the conclusions of this article will be made available by the authors, without undue reservation.

\section{REFERENCES}

Almeida, O. G. G., Pinto, U. M., Matos, C. B., Frazilio, D. A., Braga, V. L., Zeska-Kressa, M. R., et al. (2020). Does quorum sensing play a role in microbial shifts along spontaneous fermentation of cocoa beans? An in silico perspective. Food Res. Int. 131:109034. doi: 10.1016/j.foodres.2020.109034

Almeida, F. A., Pinto, U. M., and Vanetti, M. C. D. (2016). Novel insights from molecular docking of SdiA from Salmonella enteritidis and Escherichia coli with quorum sensing and quorum quenching molecules. Microb. Pathog. 99, 178-190. doi: 10.1016/j.micpath.2016.08.024

Almeida, F. A., Vargas, E. L. G., Carneiro, D. G., Pinto, U. M., and Vanetti, M. C. D. (2018). Virtual screening of plant compounds and nonsteroidal anti-inflammatory drugs for inhibition of quorum sensing and biofilm formation in Salmonella. Microb. Pathog. 121, 369-388. doi: 10.1016/j.micpath.2018.05.014

Aswathanarayan, J. B., and Vittal, R. R. (2018). Inhibition of biofilm formation and quorum sensing mediated phenotypes by berberine in Pseudomonas aeruginosa and Salmonella typhimurium. RSC Adv. 8, 36133-36141. doi: 10.1039/C8RA06413J

Ayaz, M., Ullah, F., Sadiq, A., Ullah, F., Ovais, M., Ahmed, J., et al. (2019). Synergistic interactions of phytochemicals with antimicrobial agents: Potential strategy to counteract drug resistance. Chem. Biol. Interact. 308, 294-303. doi: 10.1016/j.cbi.2019.05.050

Bai, A. J., and Rai, V. R. (2011). Bacterial quorum sensing and food industry. Compr. Rev. Food Sci. Food Saf. 10, 183-193. doi: 10.1111/j.1541-4337.2011.00150.x

Bali, E. B., Türkmen, K. E., Erdönmez, D., and Saglam, N. (2019). Comparative study of inhibitory potential of dietary phytochemicals against quorum sensing activity of and biofilm formation by Chromobacterium violaceum 12472, and swimming and swarming behaviour of Pseudomonas aeruginosa PAO1. Food Technol. Biotechnol. 57, 212-221. doi: 10.17113/ftb.57.02.19.5823

Barreca, D., Bellocco, E., Laganá, G., Ginestra, G., and Bisignano, C. (2014). Biochemical and antimicrobial activity of phloretin and its glycosilated derivatives present in apple and kumquat. Food Chem. 160, 292-297. doi: 10.1016/j.foodchem.2014.03.118

Basith, S., Cui, M., Hong, S., and Choi, S. (2016). Harnessing the therapeutic potential of capsaicin and its analogues in pain ans other diseases. Molecules 21:966. doi: 10.3390/molecules21080966

Borges, A., Ferreira, C., Saavedra, M. J., and Simões, M. (2013). Antibacterial activity and mode of action of ferulic and gallic acids against pathogenic bacteria. Microb. Drug Resist. 19, 256-265. doi: 10.1089/mdr.2012.0244

\section{AUTHOR CONTRIBUTIONS}

All authors have made direct and intellectual contribution to the work and approved it for publication.

\section{FUNDING}

We thank São Paulo Research Foundation (FAPESP 2013/07914-8) and National Council for Scientific and Technological Development (CNPq-Brazil-grant \#422242/2018-7 and CNPQ 430439/2018-0) for financial support. We acknowledge QIAGEN Company for the license to use the CLC Drug Discovery Workbench 4.0 software. CS and EL acknowledge CNPq-Brazil and the Coordination for the Improvement of Higher Education Personnel (CAPES-Brazil) for scholarships.

\section{SUPPLEMENTARY MATERIAL}

The Supplementary Material for this article can be found online at: https://www.frontiersin.org/articles/10.3389/fmicb.2021.735931/ full\#supplementary-material

Borges, A., Serra, S., Abreu, A. C., Saavedra, M. J., Salgado, A., and Simões, M. (2014). Evaluation of the effects of selected phytochemicals on quorum sensing inhibition and in vitro cytotoxicity. Biofouling 30, 183-195. doi: 10.1080/08927014.2013.852542

Brackman, G., Hillaert, U., Van Calenbergh, S., Nelis, H. J., and Coenye, T. (2009). Use of quorum sensing inhibitors to interfere with biofilm formation and development in Burkholderia multivorans and Burkholderia cenocepacia Res. Microbiol. 160, 144-151. doi: 10.1016/j.resmic.2008.12.003

Cannatelli, A., Principato, S., Colavecchio, O. L., Pallecchi, L., and Rossolini, G. M. (2018). Synergistic activity of colistin in combination with resveratrol against colistin-resistant gram-negative pathogens. Front. Microbiol. 9:1808. doi: 10.3389/fmicb.2018.01808

Carette, J., Nachtergael, A., Duez, P., Jaziri, M., and Rasamiravaka, T. (2020). "Natural compounds inhibiting Pseudomonas aeruginosa biofilm formation by targeting quorum sensing circuitry," in Bacterial Biofilms. eds. S. Dincer, M. S. Özdenefe, and A. Arkut (London, UK: IntechOpen).

Chen, C., Schauder, S., Potier, N., Dorsselaer, A. V., Pelczer, I., Bassler, B. L., et al. (2002). Structural identification of a bacterial quorum sensing signal containing boron. Nature 415, 545-549. doi: 10.1038/415545a

Chen, G., Swem, L. R., Swem, D. L., Stauff, D. L., O’Loughlin, C. T., Jeffrey, P. D. et al. (2011). A strategy for antagonizing quorum sensing. Mol. Cell 42, 199-209. doi: 10.1016/j.molcel.2011.04.003

Cho, H. S., Lee, J. H., Ryu, S. Y., Joo, S. W., Cho, M. H., and Lee, J. (2013) Inhibition of Pseudomonas aeruginosa and Escherichia coli O157:H7 biofilm formation by plant metabolite $\varepsilon$-Viniferin. J. Agric. Food Chem. 61, 7120-7126. doi: 10.1021/jf4009313

CLSI - Clinical and Laboratory Standards Institute (2007). Performance Standards for Antimicrobial Susceptibility Testing: Seventeenth Informational Supplement. Wayne, PA: CLSI.

Defoirdt, T., Brackman, G., and Coenye, T. (2013). Quorum sensing inhibitors: how strong is the evidence? Trends Microbiol. 21, 619-624. doi: 10.1016/j. tim.2013.09.006

Ding, T., Li, T., and Li, J. (2018). Impact of curcumin liposomes with antiquorum sensing properties against foodborne pathogens Aeromonas hydrophila and Serratia grimesii. Microb. Pathog. 122, 137-143. doi: 10.1016/j. micpath.2018.06.009 
Ding, T., Li, T., Wang, Z., and Li, J. (2017). Curcumin liposomes interfere with quorum sensing of Aeromonas sobria and in silico analysis. Sci. Rep. 7:8612. doi: 10.1038/s41598-017-08986-9

Duarte, A., Alves, A. C., Ferreira, S., Silva, F., and Domingues, F. C. (2015). Resveratrol inclusion complexes: antibacterial and anti-biofilm activity against Campylobacter spp. and Arcobacter butzleri. Food Res. Int. 77, 244-250. doi: 10.1016/j.foodres.2015.05.047

Grandclément, C., Tannières, M., Moréra, S., Dessaux, Y., and Faure, D. (2016). Quorum quenching: role in nature and pplied developments. FEMS Microbiol. Rev. 40, 86-116. doi: 10.1093/femsre/fuv038

Gutiérrez-Del-Rio, I., Fernández, J., and Lombó, F. (2018). Plant nutraceuticals as antimicrobial agents in food preservation: terpenoids, polyphenols and thiols. Int. J. Antimicrob. Agents 52, 309-315. doi: 10.1016/j. ijantimicag.2018.04.024

Jimenez, P. N., Koch, G., Papaicannou, E., Wahjudi, M., Krzesiak, J., Coenye, T., et al. (2010). Role of PvdQ in Pseudomonas aeruginosa virulence under iron-limiting conditions. Microbiology 156, 49-59. doi: 10.1099/mic.0.030973-0

Kali, A., Bhuvaneshwar, D., Charles, P. M. V., and Seetha, K. S. (2016). Antibacterial synergy of curcumin with antibiotics against biofilm producing clinical bacterial isolates. J. Basic Clin. Pharm. 7, 93-96. doi: 10.4103/0976-0105.183265

Kim, C. S., Gatsios, A., Cuesta, S., Lam, Y. C., Wei, Z., Chen, H., et al. (2020). Characterization of autoinducer-3 structure and biosynthesis in E. coli. ACS Cent. Sci. 6, 197-206. doi: 10.1021/acscentsci.9b01076

LaSarre, B., and Federle, M. J. (2013). Exploiting quorum sensing to confuse bacterial pathogens. Microbiol. Mol. Biol. Rev. 77, 73-111. doi: 10.1128/ MMBR.00046-12

Lee, J. H., Cho, H. S., Joo, S. W., Regmi, S. C., Kim, J. A., Ryu, C. M., et al. (2013). Diverse plant extracts and trans-resveratrol inhibit biofilm formation and swarming of Escherichia coli O157:H7. Biofouling 29, 1189-1203. doi: 10.1080/08927014.2013.832223

Lima, E. M. F., Quecán, B. X. V., Cunha, L. R., Franco, B. D. G. M., and Pinto, U. M. (2020). "Cell-cell communication in lactic acid bacteria," in Lactic Acid Bacteria A Functional Approach. eds. M. A. C. Albuquerque, A. M. Leblanc, J. G. Leblanc and R. Bedani (Boca Raton: CRC Press), 1-14.

Liu, L., Beck, C., Nohr-Meldgaard, K., Peschel, A., Kretschmer, D., Ingmer, H., et al. (2020a). Inhibition of the ATP synthase sensitizes Staphylococcus aureus toward human antimicrobial peptides. Sci. Rep. 10:11391. doi: 10.1038/ s41598-020-68146-4

Liu, L., Yu, J., Shen, X., Cao, X., Zhan, Q., Guo, Y., et al. (2020b). Resveratrol enhances the antimicrobial effect of polymyxin B on Klebsiella pneumonia and Escherichia coli isolates with polymycin B resistance. BMC Microbiol. 20:306. doi: 10.1186/s12866-020-01995-1

Mangoudehi, H. T., Zamani, H., Shahangian, S. S., and Mirzanejad, L. (2020). Effect of curcumin on the expression of ahyI/R quorum sensing genes and some associated phenotypes in pathogenic Aeromonas hydrophila fish isolares. World J. Microbiol. Biotechnol. 36:70. doi: 10.1007/s11274-020-02846-x

Martins, M. L., Pinto, U. M., Riedel, K., and Vanetti, M. C. D. (2018). Quorum sensing and spoilage potential of psychrotrophic Enterobacteriaceae isolated from milk. Biomed. Res. Int. 2018:2723157. doi: 10.1155/2018/2723157

Maura, D., Ballok, A. E., and Rahme, L. G. (2016). Considerations and caveats in antivirulence drug development. Curr. Opin. Microbiol. 33, 41-46. doi: 10.1016/j.mib.2016.06.001

Mion, S., Rémy, B., Plener, L., Brégeon, F., Chabrière, E., and Daudé, D. (2019). Quorum quenching lactonase strengthens bacteriophage and antibiotic arsenal against Pseudomonas aeruginosa clinical isolates. Front. Microbiol. 10:2049. doi: $10.3389 /$ fmicb.2019.02049

Monnet, V., Juillard, V., and Gardan, R. (2014). Peptide conversations in Grampositive bacteria. Crit. Rev. Microbiol. 43, 339-351. doi: 10.3109/1040841X.2014.948804

Monte, D. F., Lincopan, N., Berman, H., Cerdeira, L., Keelara, S., Thakur, S., et al. (2019). Genomic features of high-priority Salmonella enterica serovars circulating in the food production chain, Brazil, 2000-2016. Sci. Rep. 9:11058. doi: 10.1038/s41598-019-45838-0

Morán, A., Gutiérrez, S., Martínez-Blanco, H., Ferrero, M. A., Monteagudo-Mera, A., and Rodríguez-Aparicio, L. B. (2014). Non-toxic plant metabolites regulate Staphylococcus viability and biofilm formation: a natural therapeutic strategy useful in the treatment and prevention of skin infections. Biofouling 30, 1175-1182. doi: 10.1080/08927014.2014.976207
Mukherjee, S., and Bassler, B. L. (2019). Bacterial quorum sensing in complex and dynamically changing environments. Nat. Rev. Microbiol. 17, 371-382. doi: 10.1038/s41579-019-0186-5

Mun, S. H., Joung, D. K., Kim, Y. S., Kang, O. H., Kim, S. B., Seo, Y. S. et al. (2013). Synergistic antibacterial effect of curcumin against methicillinresistant Staphylococcus aureus. Phytomedicine 20, 714-718. doi: 10.1016/j. phymed.2013.02.006

Nazir, N., Nisar, M., Ahmad, S., Wadood, S. F., Jan, T., Zahoor, M., et al. (2020). Characterization of phenolic compounds in two novel lines of Pisum sativum L. along with their in vitro antioxidant potential. Environ. Sci. Pollut. Res. 27, 7639-7646. doi: 10.1007/s11356-019-07065-y

Nazzaro, F., Fratianni, F., and Coppola, R. (2013). Quorum sensing and phytochemicals. Int. J. Mol. Sci. 14, 12607-12619. doi: 10.3390/ijms140612607

O'May, C., and Tufenkji, N. (2011). The swarming motility of Pseudomonas aeruginosa is blocked by cranberry proanthocyanidins and other tannincontaining materials. Appl. Environ. Microbiol. 77, 3061-3067. doi: 10.1128/ AEM.02677-10

Packiavathy, I. A. S. V., Priya, S. S. K. P., and Ravi, A. V. (2014). Inhibition of biofilm development of uropathogens by curcumin - an anti-quorum sensing agent from Curcuma longa. Food Chem. 148, 453-460. doi: 10.1016/j. foodchem.2012.08.002

Qin, N., Tan, X., Jiao, Y., Liu, L., Zhao, W., Yang, S., et al. (2014). RNA-Seqbased transcriptome analysis of methicillin-resistant Staphylococcus aureus biofilm inhibition by ursolic acid and resveratrol. Sci. Rep. 4:5467. doi: 10.1038/srep05467

Quecán, B. X. V., Rivera, M. L. C., and Pinto, U. M. (2018). "Bioactive phytochemicals targeting microbial activities mediated by quorum sensing," in Biotechnological Applications of Quorum Sensing Inhibitors. ed. V. Kalia (Singapore: Springer).

Quecán, B. X. V., Santos, J. T. S., Rivera, M. L. C., Hassimoto, N. M. A., Almeida, F. A., and Pinto, U. M. (2019). Effect of quercetin rich onion extracts on bacterial quorum sensing. Front. Microbiol. 10:867. doi: 10.3389/ fmicb.2019.00867

Rivera, M. L. C., Hassimotto, N. M. A., Bueris, V., Sircili, M. P., Almeida, F. A., and Pinto, U. M. (2019). Effect of Capsicum frutescens extract, capsaicin, and luteolin on quorum sensing regulated phenotypes. J. Food Sci. 84, 1477-1486. doi: 10.1111/1750-3841.14648

Rütschlin, S., and Böttcher, T. (2019). Inhibitors of bacterial swarming behavior. Chemistry 26, 964-979. doi: 10.1002/chem.201901961

Saeki, E. K., Kobayashi, R. K. T., and Nakazato, G. (2020). Quorum sensing system: Target to control the spread of bacterial infections. Microb. Pathog 142:104068. doi: 10.1016/j.micpath.2020.104068

Sanhueza, L., Melo, R., Montero, R., Maisey, K., Mendoza, L., and Wilkens, M. (2017). Synergistic interations between phenolic compounds identified in grape pomace extract with antibiotic of different classes against Staphylococcus aureus and Escherichia coli. PLoS One 12:e0172273. doi: 10.1371/journal. pone. 0172273

Santos, C. A., Almeida, F. A., Quecán, B. X. V., Pereira, P. A. P., Gandra, K. M. B. Cunha, L. R., et al. (2020). Bioactive properties of Syzygium cumini (L.) skeels pulp and seed phenolic extracts. Front. Microbiol. 11:990. doi: 10.3389/ fmicb.2020.00990

Silva, V., Igrejas, G., Falco, V., Santos, T. P., Torres, C., Oliveira, A. M. P., et al. (2018). Chemical composition, antioxidante and antimicrobial activity of phenolic compounds extracted from wine industry by-products. Food Control 92, 516-522. doi: 10.1016/j.foodcont.2018.05.031

Singh, V. K., Mishra, A., and Jha, B. (2019). 3-benzyl-hexahydro-pyrrolo[1,2-a] pyrazine-1,4-dione extracted from exiguobacterium indicum showed antibiofilm activity against Pseudomonas aeruginosa by attenuating quorum sensing. Front. Microbiol. 10:1269. doi: 10.3389/fmicb.2019.01269

Sorrentino, E., Succi, M., Tipaldi, L., Pannella, G., Maiuro, L., Sturchio, M., et al. (2018). Antimicrobial activity of gallic acid against food-related Pseudomonas strains and its use as biocontrol tool to improve the shelf life of fresh black truffles. Int. J. Food Microbiol. 266, 183-189. doi: 10.1016/j. ijfoodmicro.2017.11.026

Vanetti, M. C. D., Carneiro, D. G., Almeida, F. A., Vargas, E. L. G., Lima, E. M. F., and Pinto, U. M. (2020). "Autoinducer-1 quorum sensing communication mechanism in Gram-negative bacteria," in Trends in Quorum Sensing and Quorum Quenching - New Perspectives and Applications. eds. V. R. Rai and J. A. Bai (Boca Raton, USA: CRC Press), 9-30. 
Vasquez-Armenta, F. J., Bernal-Mercado, A. T., Tapia-Rodriguez, M. R., Gonzalez-Aguilar, G. A., Lopez-Zavala, A. A., Martinez-Tellez, M. A., et al. (2018). Quercetin reduces adhesion and inhibits biofilm development by Listeria monocytogenes by reducing the amount of extracellular proteins. Food Control 90, 266-273. doi: 10.1016/j.foodcont.2018.02.041

Vaughn, A. R., Haas, K. N., Burney, W., Andersen, E., Clark, A. K., Crawford, R., et al. (2017). Potential role of curcumin against biofilm-producing organisms on the skin: a review. Phytother. Res. 31, 1807-1816. doi: 10.1002/ptr.5912

Vipin, C., Saptami, K., Fida, F., Mujeeburahiman, M., Rao, S. S., Athmika, A., et al. (2020). Potential synergistic activity of quercetin with antibiotics against multidrug-resistant clinical strains of Pseudomonas aeruginosa. PLoS One 15:e0241304. doi: 10.1371/journal.pone.0241304

WHO - World Health Organization (2018). High levels of antibiotic resistance found worldwide new data shows. Available at: https://www.who.int/newsroom/detail/29-01-2018-high-levels-of-antibiotic-resistance-found-worldwidenew-data-shows (Accessed February 07, 2021).

Wiegand, I., Hilpert, K., and Hancock, R. E. W. (2008). Agar and broth dilution methods to determine the minimal inhibitory concentration (MIC) of antimicrobial substance. Nat. Protoc. 3, 163-175. doi: 10.1038/ nprot.2007.521

Xu, Q., Barrios, C. A., Cutright, T., and Zhang, N. B. M. (2005). Evaluation of toxicity of capsaicin and zosteric acid and their potential application as antifoulants. Environ. Toxicol. 20, 467-474. doi: 10.1002/tox.20134

Yang, H., Cheng, J., Hu, L., Zhu, Y., and Li, J. (2012). Mechanisms of antimicrobial resistance in Serratia marcescens. Afr. J. Microbiol. Res. 6, 4427-4437. doi: 10.5897/AJMR11.1545

Zhang, W., and Li, C. (2015). Exploiting quorum sensing interfering strategies in Gram-negative bacteria for the enhancement of environmental applications. Front. Microbiol. 6:1535. doi: 10.3389/fmicb.2015.01535

Zhang, C., Zhu, S., Jatt, A. N., and Zeng, M. (2016). Characterization of N-acyl homoserine lactones (AHLs) producing bactéria isolated from vacum-packaged refrigerated turbot (Scophthalmus maximus) and possible influence of exogenous
AHLs on bacterial phenotype. J. Gen. Appl. Microbiol. 62, 60-67. doi: 10.2323/ jgam.62.60

Zheng, D., Huang, C., Huang, H., Zhao, Y., Khan, M. R. U., Zhao, H., et al. (2020). Antibacterial mechanism of curcumin: a review. Chem. Biodivers. 17:e2000171. doi: 10.1002/cbdv.202000171

Zhou, J. W., Chen, T. T., Tan, X. J., Sheng, J. Y., and Jia, A. Q. (2018). Can the quorum sensing inhibitor resveratrol function as an aminoglycoside antibiotic accelerant against Pseudomonas aeruginosa. Int. J. Antimicrob. Agents 52, 35-41. doi: 10.1016/j.ijantimicag.2018.03.002

Zhou, Y., Guan, X., Zhu, W., Liu, Z., Wang, X., Yu, H., et al. (2014). Capsaicin inhibits Porphyromonas gingivalis growth, biofilm formation, gingivomucosal inflammatory cytokine secretion, and in vitro osteoclastogenesis. Eur. J. Clin. Microbiol. Infect. Dis. 33, 211-219. doi: $10.1007 /$ s10096-013-1947-0

Conflict of Interest: The authors declare that the research was conducted in the absence of any commercial or financial relationships that could be construed as a potential conflict of interest.

Publisher's Note: All claims expressed in this article are solely those of the authors and do not necessarily represent those of their affiliated organizations, or those of the publisher, the editors and the reviewers. Any product that may be evaluated in this article, or claim that may be made by its manufacturer, is not guaranteed or endorsed by the publisher.

Copyright (c) 2021 Santos, Lima, Franco and Pinto. This is an open-access article distributed under the terms of the Creative Commons Attribution License (CC BY). The use, distribution or reproduction in other forums is permitted, provided the original author(s) and the copyright owner(s) are credited and that the original publication in this journal is cited, in accordance with accepted academic practice. No use, distribution or reproduction is permitted which does not comply with these terms. 